\title{
Natural history of treatment of facial dyskinesias with botulinum toxin: a study of 50 consecutive patients over seven years
}

\author{
Joseph A Mauriello, John Aljian
}

\begin{abstract}
To determine the long-term efficacy of botulinum toxin injections for the treatment of facial dyskinesias we studied $\mathbf{5 0}$ consecutive patients with blepharospasm, hemifacial spasm, and Meige syndrome. All received their first injection between September 1983 and June 1984. A total of 520 injections were given; the average number of injections per patient was 10.4 over the seven-year period ending September 1990. Twenty-six (52\%) of the patients continued to return for periodic injections, while three patients no longer receive injections since they failed to respond adequately to treatment. Three patients with blepharospasm were in remission and required no further treatment, after a series of six, four, and three injections. Six patients were treated until they died of causes unrelated to facial dyskinesia or its treatment. Six patients are still being treated elsewhere because they could obtain injections closer to their homes. Five of the original 50 patients have been lost to follow-up. A patient with hemifacial spasm had one injection with good result but was not sufficiently bothered by her disease to return for reinjection. Complications were transient, minimal, well tolerated, and did not increase with increased number of injections.
\end{abstract}

Several studies have established the relatively short-term efficacy of botulinum toxin (Oculinum injections) in the treatment of blepharospasm, hemifacial spasm, and Meige syndrome. ${ }^{1-15}$ However, the long-term acceptance by patients of this treatment has not been as well documented. ${ }^{16-18}$ In Kraft and Lang's study, the follow-up period for 76 patients who were treated with Oculinum injections was 10 to 36 months. ${ }^{16}$ In Elston's series of 101 patients the mean follow-up period was 26.7 months with a range of 1 to 51 months. ${ }^{17}$ Dutton and Buckley's study of 230 patients included 30 patients who had 10 or more treatments over a 51 -month follow-up period. ${ }^{18}$ We reviewed the first 50 consecutive patients of a total series of 302 treated to date in order to understand the natural course of facial dyskinesias treated with botulinum toxic type A over a seven-year period. The minimum follow-up period for all patients was 75 months.

\section{Patients and methods}

Charts from the first 50 consecutive patients with blepharospasm, hemifacial spasm, and Meige syndrome treated with botulinum toxin injection were reviewed. Data analysed included age of onset, dosage, number of injections, treatment additional to botulinum injections, and complications. Patients no longer treated were contacted by telephone. All 50 patients were initially treated between September 1983 and June 1984. Of these 50 patients 31 were female and 19 were male. Thirty-three patients had blepharospasm, 13 had hemifacial spasm, and four had Meige syndrome (Table 1). The examination and injection techniques have been previously described. ${ }^{513}$ All patients received the American product of botulinum toxin, Oculinum, rather than the British product, Dysport.

The initial treatment consisted of 5 units of botulinum toxic injected in divided doses into the medial and lateral portions of the pretarsal orbicularis muscle of the upper eyelids, and 5 units into the corresponding medial and lateral parts of the lower eyelids; and additional 2.5 units was injected into the lateral canthus. Patients with brow spasms received 5 units in two separate injections into the procerus muscle of the medial brow. Patients with facial spasms were given 5 units in two separate injections into the cheek area just below the malar eminence after having received eyelid injections. When examined two weeks after injection the patients with a poor response received an additional 10 units (double dose) in each upper lid.

\section{Results}

Fifty patients received a total of 520 injections between September 1983 and September 1990. The mean age of the patients at the onset of their disease was $56 \cdot 5$ years (range 37-76) for blepharospasm, 62.5 years (range 40-76) for hemifacial spasm, and $72 \cdot 3$ years (range 52-64) for Meige syndrome. The average number of injections per patient was 10.4 over the sevenyear period.

Of the original 50 patients $26(52 \%)$ continued to return for periodic injections. The patients who continued to receive injections included 18 of 33 patients with blepharospasm, seven of 13 patients with hemifacial spasm, and one of four patients with Meige syndrome. The mean number of injections was $15 \cdot 3$ for 18 patients with blepharospasm, $11 \cdot 3$ injections for seven

Table 1 Sex distribution by diagnosis of 50 patients

\begin{tabular}{lccc}
\hline & $\begin{array}{l}\text { No. of } \\
\text { patients }\end{array}$ & Male & Female \\
\hline Blepharospasm & $33(66 \%)$ & $12(32 \cdot 2 \%)$ & $21(67 \cdot 8 \%)$ \\
Hemifacial spasm & $13(26 \%)$ & $5(28 \cdot 5 \%)$ & $8(61 \cdot 5 \%)$ \\
Meige syndrome & $4(8 \%)$ & $2(50 \%)$ & $2(50 \%)$ \\
\hline
\end{tabular}


Table 2 Follow-up data on 50 patients by diagnosis

\begin{tabular}{llllc}
\hline Treatment status & Blepharospasm & Hemifacial spasm & Meige syndrome & Total \\
\hline Still treated & 19 & 6 & 1 & $26(52 \%)$ \\
Treatment no longer required & 3 & 0 & 0 & $3(6 \%)$ \\
Treatment failures & 1 & 1 & 1 & $3(6 \%)$ \\
Treated until died & 1 & 4 & 1 & $6(12 \%)$ \\
Treated elsewhere & 4 & 1 & 1 & $6(12 \%)$ \\
Lost to follow-up & 5 & 0 & 0 & $5(10 \%)$ \\
Good treatment response but & 0 & 1 & 0 & $1(2 \%)$ \\
no longer injected & & & & \\
\hline
\end{tabular}

patients with hemifacial spasm, and 11.3 for the patient with Meige syndrome. The mean duration of action of the injections was 14.9 weeks for 18 patients with blepharospasm, 16.3 weeks for seven patients with hemifacial spasm, and 11 weeks for the one patient with Meige syndrome.

Three patients no longer receive injections since they failed to repond to periodic injections of botulinum. One such patient with blepharospasm responded to the initial injection but failed to respond to subsequent injections on five separate occasions at higher than standard dose levels. A second patient with Meige syndrome had only a partial response to initial injection and did not return for further treatment. A third patient, a 40-year-old male with left-sided hemifacial spasm, was treated from January 1984 to May 1987. The first seven of 10 treatments produced excellent results that lasted 14 to 18 weeks. The last three injections lasted 10 , seven, and four weeks respectively. A neurosurgical consultation was obtained and in October 1987, he underwent a left retromastoid craniotomy with microvascular decompression of the seventh nerve, with good result. A third patient with blepharospasm received one injection and did not improve; she did not wish to return for reinjection.

Three patients with blepharospasm required no further treatment after a series of six, four, and three injections, respectively. Two of these patients have been in remission for over six years and the third patient for over five years.

Six patients were treated until they died of causes unrelated to facial dyskinesia or its treatment. Six patients are still being treated elsewhere because they could obtain injections closer to their homes. Five of the original 50 patients have been lost to follow-up. A patient with hemifacial spasm had one injection with good result but was not sufficiently bothered by her disease to return for reinjection (Table 2).

Six patients who continue to be treated had excision of excess skin with plication of the levator aponeurosis without effect on the duration of the injections. One patient with hemifacial spasm developed a basal cell carcinoma of the lower lid which was excised; another patient with hemifacial spasm had a marginal rotation of the right upper lid prior to injections. The duration of effect of the injection was not influenced by the surgery. These eight patients continued to be treated at expected intervals and received a mean of $14 \cdot 1$ injections without diminution of effect.

Six of 50 patients, all with blepharospasm, received a double dose in the upper lids (10 units) in each upper lid, and one patient received a quadruple dose ( 20 units) in the upper lid. Twenty-four of 50 patients received bilateral brow injections ( 5 units in each brow). Four patients with Meige syndrome received facial injections that varied in dosage from 5 to 20 units; one patient received 10 units in the masseter muscle for jaw spasms. All such patients also received eyelid injections. Three of the patients with hemifacial spasm received 5 units in the cheek.

Two of the patients who continue to receive injections are concurrently administered minor tranquillisers by their physician.

No complications, transient or mild, increased with the number of injections. In addition no patient withdrew from treatment owing to complications. ${ }^{613}$ Tearing, the most common complication, was either due to weakness of the lacrimal pump or was reflex in nature due to lagophthalmos or to pre-existing dry eye (18 patients or $36 \%$ ). The latter group of patients often had a concomitant foreign body sensation in the involved eyes. Tearing generally lasted one to two weeks and rarely up to six weeks from the time of injection; symptoms were relieved by topical lubricants and by increased voluntary blinking. Other complications were mild ptosis (seven patients or 14\%), ectropion (one patient or $2 \%$ ), entropion (one patient or $2 \%$ ), and double vision (one patient or $2 \%$ ). The patient with lower lid ectropion had hemifacial spasm; recurrent ectropion was prevented by injecting one-half the usual dose ( $2 \frac{1}{2} 2$ units) into the lower lid. The patient with double vision had received a double dose of Oculinum in the upper lid; he had temporary diplopia in upgaze in four of eight injections; the double dose was necessary for a duration of effect of 10 to 11 weeks.

\section{Discussion}

The short-term effectiveness of botulinum toxin type A injection for the control of facial dyskinesias, including benign essential blepharospasm, hemifacial spasm, and Meige syndrome, has been well documented in several studies. ${ }^{1-15}$ The percentage of patients who respond has varied from $93 \%{ }^{16}$ to $97 \% .^{13}$ The study with longest follow-up period consisted of a cohort of 30 of a total of 230 patients who had 10 or more treatments over a 51 -month follow-up period. ${ }^{18}$ The natural history of a group of patients who have had botulinum toxin injections over a long period has not been elucidated.

The present study covered 50 consecutive patients with a minimum follow-up of over 75 months. The relatively high proportion of patients $(52 \%)$ that continue to receive injections over the seven-year period strongly supports the debilitating nature of the facial dyskinesias, the patient's need for treatment, and the efficacy of botulinum toxin.

The low number of long-term failures, three of 50 patients, is again consistent with other studies with shorter follow-up. One of the three patients failed to respond after having had seven prior successful injections, and the other two patients responded to the initial injections but failed to respond to subsequent treatments. These findings suggest that clinically active antibodies 
rarely develop to the toxin, at least over a sevenyear period. The data show that, if a given patient responds to two or more injections, tolerance to the drug will be unlikely to develop subsequently. Possibly longer durations of treatment and higher doses used in the treatment of other conditions such as torticollis may lead to tolerance. ${ }^{19}$ Antibody studies are worth undertaking. Our study provides no evidence that the orbicularis muscle weakens from repeated injections over time to the extent that lower dosages of toxin are effective.

As in studies with shorter follow-up periods, complications were all transient, minimal, well tolerated, and did not increase with increased number of injections. These complications included tearing, ptosis, and rarely ectropion, entropion, and double vision.

Eight of the patients had eyelid surgery, including plication of the levator aponeurosis and repair of dermatochalasis (six patients), marginal rotation of the upper lid (one patient), and excision of a basal cell carcinoma of the lower lid (one patient). These eight patients continued to be treated at expected intervals and received a mean of $14 \cdot 1$ injections without diminution of effect. Our findings suggest that eyelid surgery may be safely performed without affecting treatment response.

Surprisingly, three of the 50 patients were in remission and no longer required treatment after a series of six, four, and three injections. To our knowledge remission after botulinum toxin injections is extremely rare and has not been previously reported. In a series of 264 patients with blepharospasm five were in remission for periods of one to six years without any treatment. ${ }^{14}$ Such remissions suggest that blepharospasm may be self-limited in rare cases with or without treatment. The possible role of the drug, if any, in causing the remission is unclear.
Supported in part by an unrestricted grant from Research to Prevent Blindness, New York, New York.

1 Jankovic J, Ford J. Blepharospasm and orofacial-cervical dystonia: clinical and pharmacologic findings in 100 patients. Ann Neurol 1983; 13: 402-11.

2 Scott AB, Kennedy RA, Stubbs HA. Botulinum A toxin injections as a treatment for blepharospasm. Arch Ophthalmol 1985; 103: 347-50.

3 Frueh BR, Musch DC. Treatment of facial spasm with botulinum toxin: an interim report. Ophthalmology 1986; 93: 917-23.

4 Shorr N, Seiff SR, Kopelman J. The use of botulinum toxin in blepharospasm. Am f Ophthalmol 1985; 99: 917-23.

5 Mauriello.JA. Blepharospasm, Meige syndrome, and hemifacial spasm: treatment with botulinum toxin. Neurology 1985; 35: 1499-500.

6 Shore JW, Leon CR, O'Connor PS, et al. Botulinum toxin for the treatment of essential blepharospasm. Ophthalmic Surg $1986 ; 17: 747-53$.

7 Engstrom PF, Arnoult JB, Mazow ML, et al. Effectiveness of botulinum toxin therapy for essential blepharospasm. Ophthalmology 1987; 94: 971-5.

8 Savino PJ, Sergott RC, Bosley TM, Schatz NJ. Hemifacial spasm treated with botulinum A toxin injection. Arch spasm treated with botulinum
Ophthalmol 1985; 103: 1305-6.

9 Dutton JJ, Buckley EG. Botulinum toxin in the management of blepharospasm. Arch Neurol 1986; 43: 380-2.

10 Cohen DA, Savino PJ, Stern MB, Hurtig HI. Botulinum injection therapy for blepharospasm: a review and report of 75 patients. Clin Neuropharmacol 1986; 9: 415-29.

11 Fahn S. The varied clinical expressions of dystonia. Neuro Clin 1984; 2: 541-54.

12 Jordan DR, Patrinely JR, Anderson RL, Thiese SM. Essential blepharospasm and related dystonias. Surv Ophthalmol 1989; 34: 123-32.

13 Mauriello JA, Coniaris H, Haupt EJ. Use of botulinum toxin in the treatment of one hundred patients with facial in the treatment of one hundred patient
dyskinesias. Ophthalmology 1987; 94: 976-9.

14 Grandas F, Elston J, Quinn N, Marsden CD. Blepharospasm a review of 264 patients. $\mathcal{F}$ Neurol Neurosurg Psychiatry 1988 51: 767-72.

15 American Academy of Ophthalmology. Botulinum toxin therapy of eye muscle disorders. Safety and effectiveness. Ophthalmology 1989; instrument and book issue: $37-41$.

16 Kraft SP, Lang AE. Botulinum toxin injections in the treatment of blepharospasm, hemifacial spasm, and eyelid fasciculations. Can F Neurol Sci 1988; 15: 276-80.

17 Elston JS. Long-term results of treatment of idiopathic blepharospasm with botulinum toxin injections. $\mathrm{Br} \mathcal{F}$ Ophthalmol 1987; 71: 664-8.

18 Dutton JJ, Buckley EG. Long-term results and complications of botulinum A toxin in the treatment of blepharospasm. Ophthalmology 1988; 95: 1529-34.

19 Jankovic J. Blepharospasm and oromandibular-laryngealcervical dystonia: a controlled trial of botulinum A toxin cervical dystonia: a controlled trial of botulinum A toxin New York: Raven Press, 1988; 50: 583-91. 